\title{
Hierarchical NiS/N-doped carbon composite hollow spheres with excellent supercapacitor performance $\uparrow$
}

Received 14th July 2017

Accepted 8th September 2017

DOI: $10.1039 / \mathrm{c} 7 \mathrm{ta06149h}$

rsc.li/materials-a

\author{
Tao Liu, ${ }^{a}$ Chuanjia Jiang, (DD ${ }^{a}$ Bei Cheng, ${ }^{a}$ Wei You (D) ac and Jiaguo Yu (D) *ab
}

Designing electrode materials with high specific capacitance is crucial for further improving the energy storage performance of supercapacitors. Nickel sulfide (NiS) nanosheets anchored on the surface of $\mathrm{N}$ doped hollow carbon spheres (NiS/NHCS) were rationally designed and synthesized via a multistep transformation approach. Uniform nickel silicate nanosheets were first deposited on the surface of NHCSs, followed by chemical conversion into NiS nanosheets. The NiS/NHCS composite shows excellent electrochemical properties as an electrode material for supercapacitors. The NiS/NHCS electrode can deliver a high specific capacitance of $1150 \mathrm{~F} \mathrm{~g}^{-1}$ at $1 \mathrm{~A} \mathrm{~g}^{-1}$ and show outstanding cycling performance with a retention rate of $76 \%$ over 4000 cycles, which are much higher than those of pure NiS hollow spheres (NiS-HS) (400 $\mathrm{F} \mathrm{g}^{-1}$ and 63\%, respectively). Moreover, a hybrid supercapacitor assembled with NiS/NHCS as the cathode and an activated carbon electrode as the anode delivers a high energy density of $38.3 \mathrm{~W} \mathrm{~h} \mathrm{~kg}^{-1}$ at a power density of $160 \mathrm{~W} \mathrm{~kg}^{-1}$ and has an excellent cycle performance with the retention rate of $96 \%$ after 5000 cycles. The exceptional electrochemical performance of the NiS/NHCS composite indicates its potential application in high-performance supercapacitors.

\section{Introduction}

Energy storage and conversion devices, in particular rechargeable batteries and supercapacitors, have attracted increasing attention for the better utilization of renewable resources. ${ }^{\mathbf{1 - 5}}$ Supercapacitors have been considered as one of the most promising device candidates due to their intriguing characteristics of fast charge/discharge capability, outstanding cycle life and high power density. ${ }^{6-10}$ Nonetheless, the relatively low energy density of supercapacitors compared with rechargeable batteries still requires significant improvement. Enhancing the specific capacitance of the electrode materials is one of the compelling approaches to solve this issue.

Recently, transition metal sulfides have been extensively investigated as novel electrode materials for supercapacitors, ${ }^{\mathbf{1 1}}$ due to their high theoretical specific capacitance, excellent

${ }^{a}$ State Key Laboratory of Advanced Technology for Materials Synthesis and Processing, Wuhan University of Technology, Luoshi Road 122, Wuhan 430070, PR China. E-mail: jiaguoyu@yahoo.com; Fax: +86-27-87879468; Tel: +86-27-87871029

${ }^{b}$ Department of Physics, Faculty of Science, King Abdulaziz University, Jeddah 21589, Saudi Arabia

'Department of Chemistry, University of North Carolina at Chapel Hill, Chapel Hill, North Carolina 27599-3290, USA

$\dagger$ Electronic supplementary information (ESI) available: Additional morphology characterizations, XPS, nitrogen adsorption-desorption data, electrochemical characterization of the NiS/N-doped carbon composite and the control samples. See DOI: $10.1039 / \mathrm{c} 7 \mathrm{ta} 06149 \mathrm{~h}$ electrical conductivity, and mechanical and thermal stability, including nickel sulfides (NiS, ${ }^{12} \mathrm{Ni}_{2} \mathrm{~S}_{3}$ (ref. 13) and $\mathrm{Ni}_{7} \mathrm{~S}_{6}$ (ref. 14)), cobalt sulfides $\left(\mathrm{Co}_{3} \mathrm{~S}_{4}\right.$ (ref. 15)), manganese sulfides $\left(\mathrm{MnS}^{16}\right)$, and binary metal sulfides $\left(\mathrm{NiCo}_{2} \mathrm{~S}_{4}\right.$ (ref. 17) and $\mathrm{MnCo}_{2} \mathrm{~S}_{4}$ (ref. 18)). Among them, nickel sulfide has received special interest. For instance, NiS box-in-box hollow structures reported by $\mathrm{Yu}$ et $a l .{ }^{19}$ delivered a high specific capacitance of $668 \mathrm{~F} \mathrm{~g}^{-1}$ at the current density of $1 \mathrm{~A} \mathrm{~g}^{-1}$ and distinguished rate performance (71\% capacitance retention at $\left.20 \mathrm{~A} \mathrm{~g}^{-1}\right)$. Yang et $a l .{ }^{20}$ synthesized hierarchical flower-like nickel sulfide which exhibited a specific capacitance of $972.3 \mathrm{~F} \mathrm{~g}^{-1}$ at $2 \mathrm{~A} \mathrm{~g}^{-1}$ and excellent cycling stability. Zhu et al. ${ }^{21}$ fabricated Nis hollow spheres via a facile template-engaged transformation approach, which showed high specific capacitances of 927-583 $\mathrm{F} \mathrm{g}^{-1}$ at different current densities of 4.08-10.2 $\mathrm{A} \mathrm{g}^{-1}$. These studies on nickel sulfide materials indicate that these unique nanostructures are able to improve the electrochemical performance for supercapacitors.

On the other hand, the growth and anchoring of functional nanomaterials on carbonaceous materials, such as graphene, $^{22-25}$ carbon nanotubes ${ }^{\mathbf{2 6}}$ and carbon fiber, ${ }^{\mathbf{1 8 , 2 7}}$ is an effective method to improve the cycling performance and rate properties of supercapacitors. ${ }^{28}$ Hollow carbon spheres (HCSs) have been regarded as an alternative substrate for preparing electrode material due to the following advantages: internal cavities serving as ion reservoirs and rapidly providing electrolyte ions for the electrochemical reactions, a carbon shell with 
good conductivity facilitating electron transport, ${ }^{29}$ and a large surface area favoring the deposition of active materials. It can therefore be expected that a thin and porous layer of active material grown on the HCS surface could exhibit enhanced energy storage capacity and rate capacity.

Herein, we reported the design and fabrication of porous NiS nanosheets grown on N-doped hollow carbon spheres (NHCSs) via an efficient template-engaged method. The first step involves fabricating $\mathrm{N}$-doped carbon spheres@SiO ${ }_{2}$ (N-carbon/ $\mathrm{SiO}_{2}$ ) with a core-shell structure, which serves as the template for the growth of nickel silicate (NiSi) nanosheets on the surface of NHCSs in the second step, yielding NiSi/NHCS hollow structures. Finally, the NiSi/NHCS hollow structure is transformed into NHCS-supported NiS nanosheets (NiS/NHCS) by hydrothermal sulfidation treatment. When used as an electrode material for supercapacitors, the NiS/NHCS composite with a hierarchically hollow structure exhibits better electrochemical properties than pure NiS hollow spheres (NiS-HS).

\section{Results and discussion}

The synthesis process of the hierarchical NiS/NHCS composite with a hollow structure is illustrated in Fig. 1. First, N-carbon/ $\mathrm{SiO}_{2}$ with uniform sizes (Fig. S1a and $\mathrm{b}^{\dagger}$ ) is synthesized via a one-step process involving the hydrolysis of tetraethoxysilane (TEOS) and the polymerization of dopamine, followed by the carbonization of the polydopamine $/ \mathrm{SiO}_{2}$ spheres. Then nickel silicate nanosheets are in situ formed on the NHCS surface via a facile hydrothermal treatment in dilute ammonia solution, where NHCSs and $\mathrm{SiO}_{2}$ cores serve as templates and the selfsacrificial substrate, respectively (step I). In this process, ammonia can not only produce an alkaline condition but also provide $\mathrm{NH}_{3}$ to coordinate with $\mathrm{Ni}^{2+} \cdot{ }^{30}$ Ammonium chloride is a necessary additive to mediate the dissociation of ammonia and inhibit the formation of nickel hydroxide. Silicate anions are released from the dissolution of the $\mathrm{SiO}_{2}$ core under alkaline conditions at high temperature. The reaction between the silicate ions and $\mathrm{Ni}^{2+}$ leads to the nucleation of $\mathrm{NiSi}$, which could preferentially deposit on the carbon shell. Continuous dissolution of the $\mathrm{SiO}_{2}$ core supplies more silicate anions for the growth of NiSi nanosheets. When there are not enough dissolved $\mathrm{Ni}^{2+}$ ions, an outer shell composed of nickel silicate nanosheets on the NHCS surface and the remaining $\mathrm{SiO}_{2}$ core

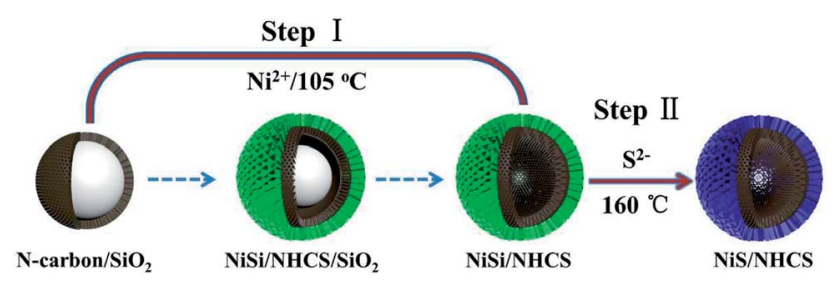

Fig. 1 Scheme for the formation of nickel sulfide (NiS) nanosheets supported on the NHCS surface by a novel conversion route: (I) growth of nickel silicate (NiSi) nanosheets on the NHCS surface with the silica core simultaneously eliminated; (II) chemical conversion of nickel silicate into NiS to form NiS/NHCS. enclosed within form a rattle-like structure ( $\mathrm{NiSi} / \mathrm{NHCS} / \mathrm{SiO}_{2}$ ). As the reaction continues, the $\mathrm{SiO}_{2}$ core is fully dissolved and a hollow nanostructure of NiSi/NHCS is eventually fabricated. Finally, the as-prepared NiSi/NHCS is transformed into NiS/ NHCS by a solution-based sulfidation approach with $\mathrm{Na}_{2} \mathrm{~S}$ as the $\mathrm{S}^{2-}$ source (step II).

The microstructures of reaction products for each stage were characterized by transmission electron microscopy (TEM). The $\mathrm{N}$-carbon $/ \mathrm{SiO}_{2}$ (Fig. 2a and e) solid spheres had a uniform diameter of $310 \mathrm{~nm}$. For NiSi/NHCS/SiO 2 obtained after reaction for $2 \mathrm{~h}$ (Fig. $2 \mathrm{~b}$ and $\mathrm{f}$ ), nickel silicate nanosheets are closely grown on the surface of NHCSs, and a gap between the NiSi/ NHCS shell and $\mathrm{SiO}_{2}$ core can be clearly observed. After $12 \mathrm{~h}$ of reaction, NiSi/NHCS (Fig. 2c and g) shows a hollow structure with a $400 \mathrm{~nm}$ outer diameter and shell thickness of $50 \mathrm{~nm}$, and this structure is maintained in the final product NiS/NHCS (Fig. 2d and h). The hollow structure combining the NiS nanosheet shell and NHCSs can provide abundant electrochemically active sites and a large electrolyte-electrode contact area for a pseudocapacitive reaction. Meanwhile, the $\mathrm{N}$-doped carbon core can boost the electron transfer, which can improve the rate capacity of supercapacitors. Additionally, the aggregation of electroactive particles and a pronounced volume variation during charge/discharge processes can be effectively inhibited due to the hollow carbon cavities.

Field-emission scanning electron microscope (FESEM) images (Fig. 3a and b) confirmed that the surface of both NiSi/NHCS and NiS/NHCS is assembled by using numerous nanosheets, and the morphology was not altered during sulfidation. The NiSi hollow sphere (NiSi-HS) (Fig. S2 $\dagger$ ) and NiS-HS (Fig. 3c) are also composed of interconnected nanosheets, and TEM images of NiS-HS (Fig. 3d) further confirm the hierarchical hollow structure. The average diameter of NiS-HS is about $380 \mathrm{~nm}$, which is smaller than that of NiS/NHCS, mainly due to the absence of the NHCS core. The $\mathrm{N}_{2}$ adsorption-desorption isotherms (Fig. S3a $\dagger$ ) of NiS/NHCS and NiS-HS can be classified as type IV with an overlap of type $\mathrm{H} 2$ and $\mathrm{H} 3$ hysteresis loops, which shows the existence of ink-bottle mesopores within the NiS nanosheets or porous NHCSs and slit-like mesopores formed between NiS nanosheets, respectively. ${ }^{31}$ NiS/NHCS and NiS-HS have hierarchical pore structures according to the wide pore size distribution (Fig. $\mathrm{S} 3 \mathrm{~b} \dagger$ ), which is beneficial for the electrolyte ion penetration..$^{32}$ Especially, the BET specific surface area and pore volume of NiS/NHCS $\left(106 \mathrm{~m}^{2} \mathrm{~g}^{-1}\right.$ and $\left.0.30 \mathrm{~cm}^{3} \mathrm{~g}^{-1}\right)$ are higher than those of NiS-HS $\left(86 \mathrm{~m}^{2} \mathrm{~g}^{-1}\right.$ and $\left.0.15 \mathrm{~cm}^{3} \mathrm{~g}^{-1}\right)$, which can provide more electroactive sites for participating in the faradaic redox reactions, leading to improved electrochemical performance.

The crystal structures of NHCSs, NiSi/NHCS and NiS/NHCS were characterized by the XRD patterns (Fig. 4a). The peak at $23.5^{\circ}$ can be assigned to graphite carbon sheets of NHCSs. In the XRD spectrum of NiSi/NHCS, the weak peaks located at $35.5^{\circ}$ and $60.7^{\circ}$ are indexed to the (200) and (060) planes of monoclinic nickel hydrosilicate, respectively. ${ }^{21}$ In the XRD spectrum of NiS/NHCS, the peaks at $19.4^{\circ}, 30.3^{\circ}, 32.7^{\circ}, 35.9^{\circ}$, $49.4^{\circ}, 53.5^{\circ}$ and $59.8^{\circ}$ can be indexed to (110), (101), (300), (021), (410), (401) and (012) crystal planes of NiS (JCPDF \#86-2281). 


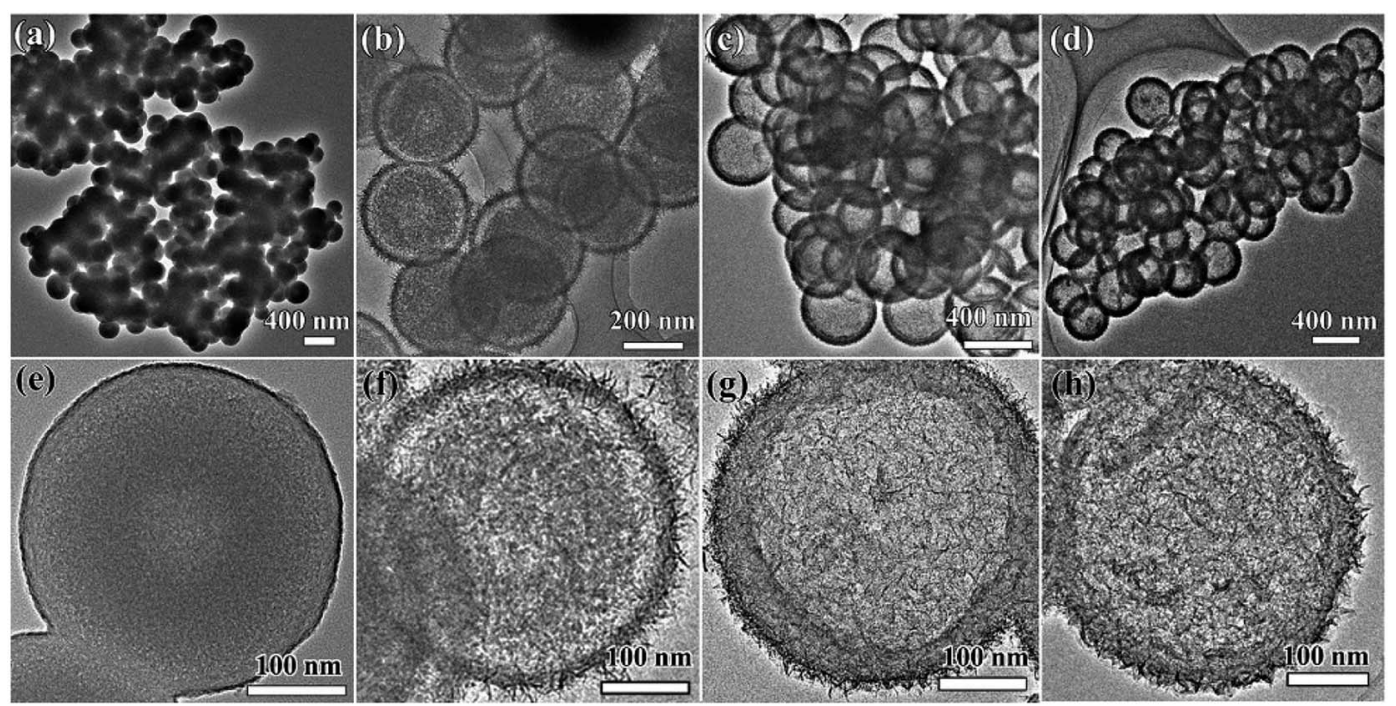

Fig. 2 TEM images of $\mathrm{N}$-carbon/SiO 2 (a and e), NiSi/NHCS/SiO $(b$ and f), NiSi/NHCS (c and g) and NiS/NHCS (d and h).

From the Raman spectra of NHCSs and NiS/NHCS (Fig. 4b), typical D and G bands at 1358 and $1596 \mathrm{~cm}^{-1}$ were observed, further confirming the existence of carbon in the NiS/NHCS composite. The relatively high intensity of the D band demonstrates the existence of substantial defects, mainly resulting from nitrogen dopants and contaminants. ${ }^{33}$ The peak at
$578 \mathrm{~cm}^{-1}$ in the spectrum of NiS/NHCS is attributed to the $\mathrm{F}_{2 \mathrm{~g}}$ model of Ni-S. ${ }^{34}$

The surface elemental composition and chemical state of $\mathrm{N}$-carbon/SiO $\mathrm{Si}_{2}$ and NiS/NHCS were analyzed by X-ray photoelectron spectroscopy (XPS). The survey XPS spectrum of $\mathrm{N}$-carbon/ $/ \mathrm{SiO}_{2}$ (Fig. S4a $\dagger$ ) demonstrates the presence of $\mathrm{C}, \mathrm{N}, \mathrm{O}$
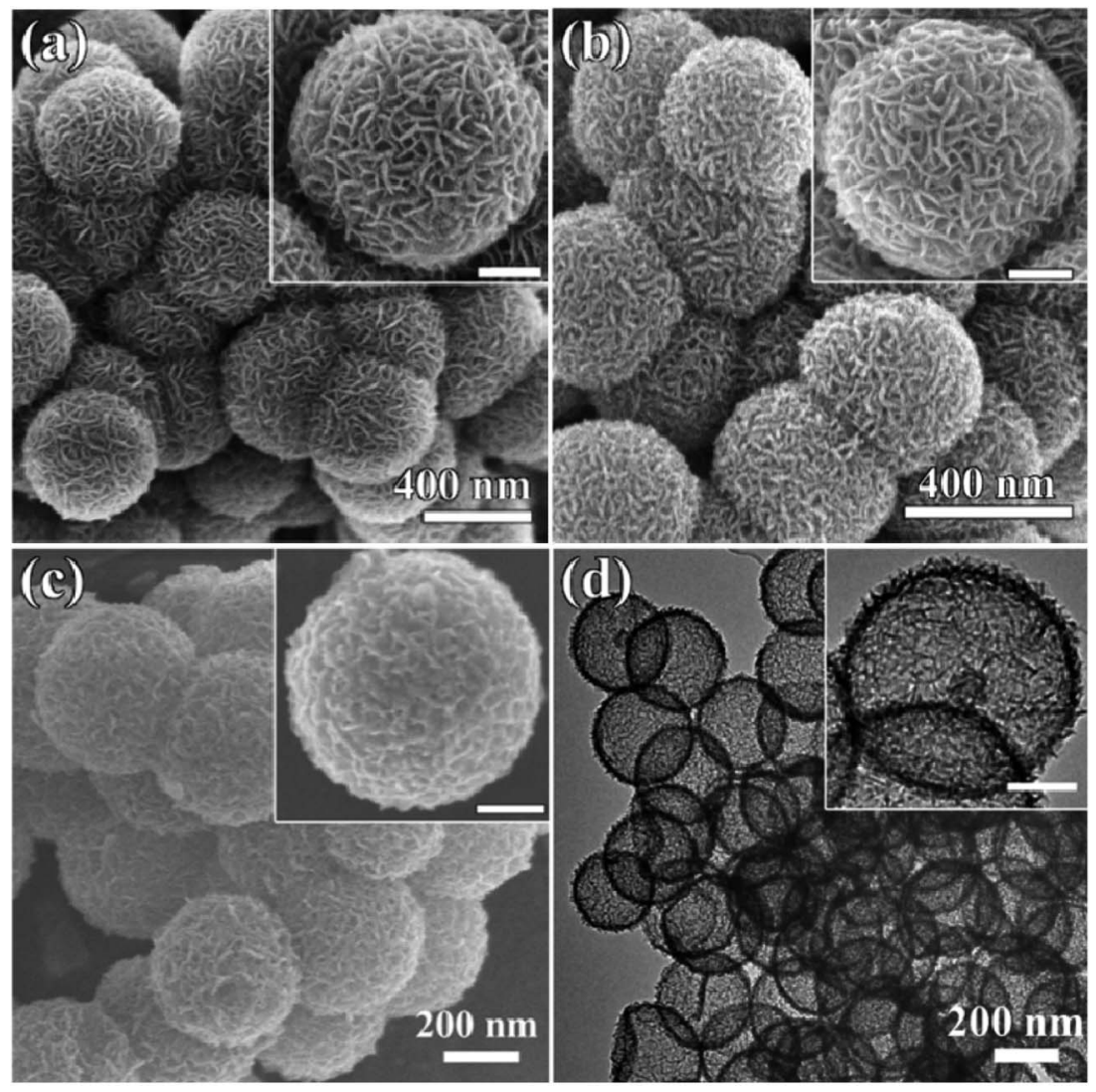

Fig. 3 FESEM images of NiSi/NHCS (a), NiS/NHCS (b) and NiS-HS (c) and the TEM image of NiS-HS (d). The scale bars of the insets are $100 \mathrm{~nm}$. 

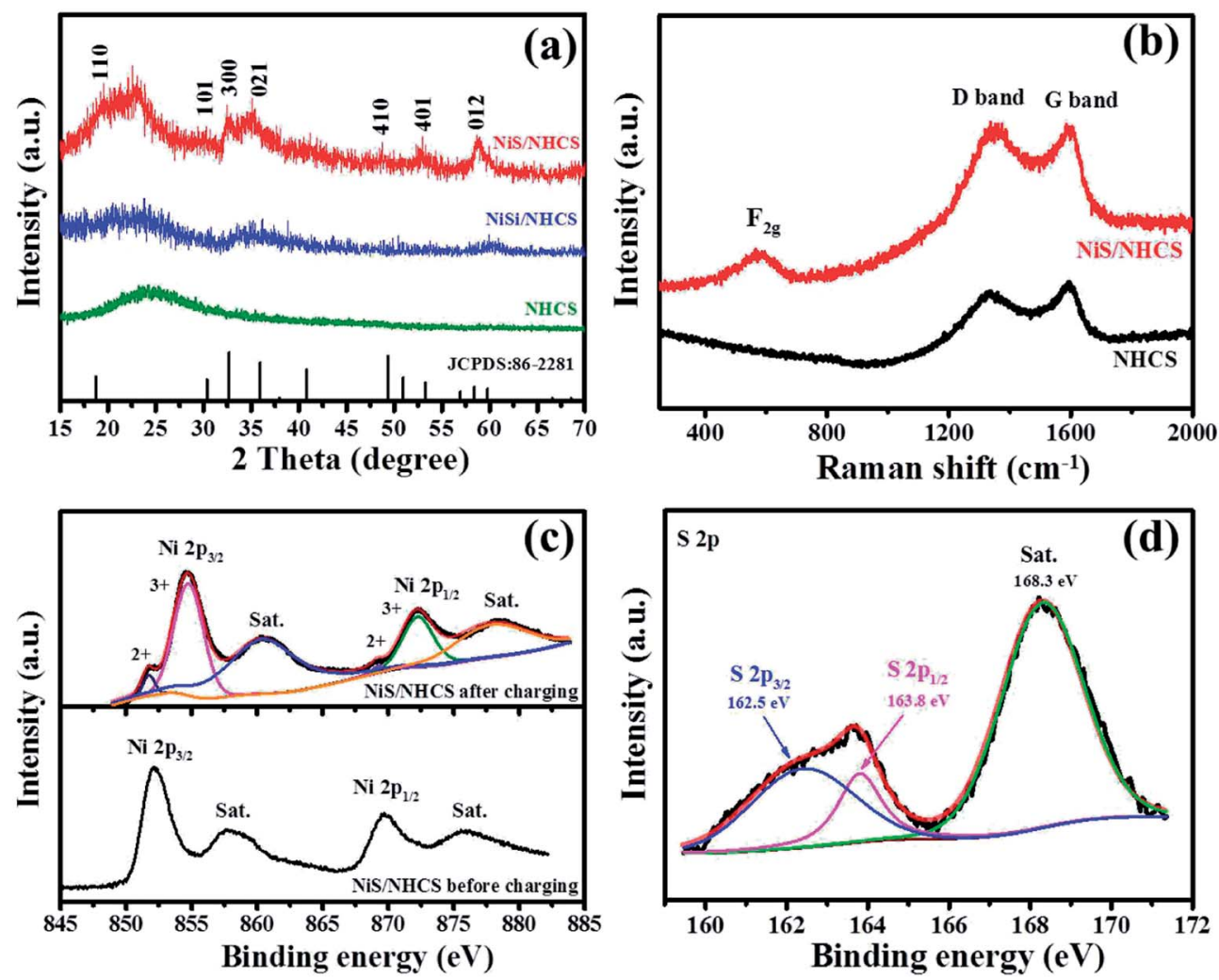

Fig. 4 (a) XRD patterns of NHCSs, NiSi/NHCS and NiS/NHCS. (b) Raman spectra of NHCSs and NiS/NHCS. High-resolution XPS spectra of Ni 2p before and after charging (c) and $\mathrm{S} 2 \mathrm{p}(\mathrm{d})$ for NiS/NHCS.
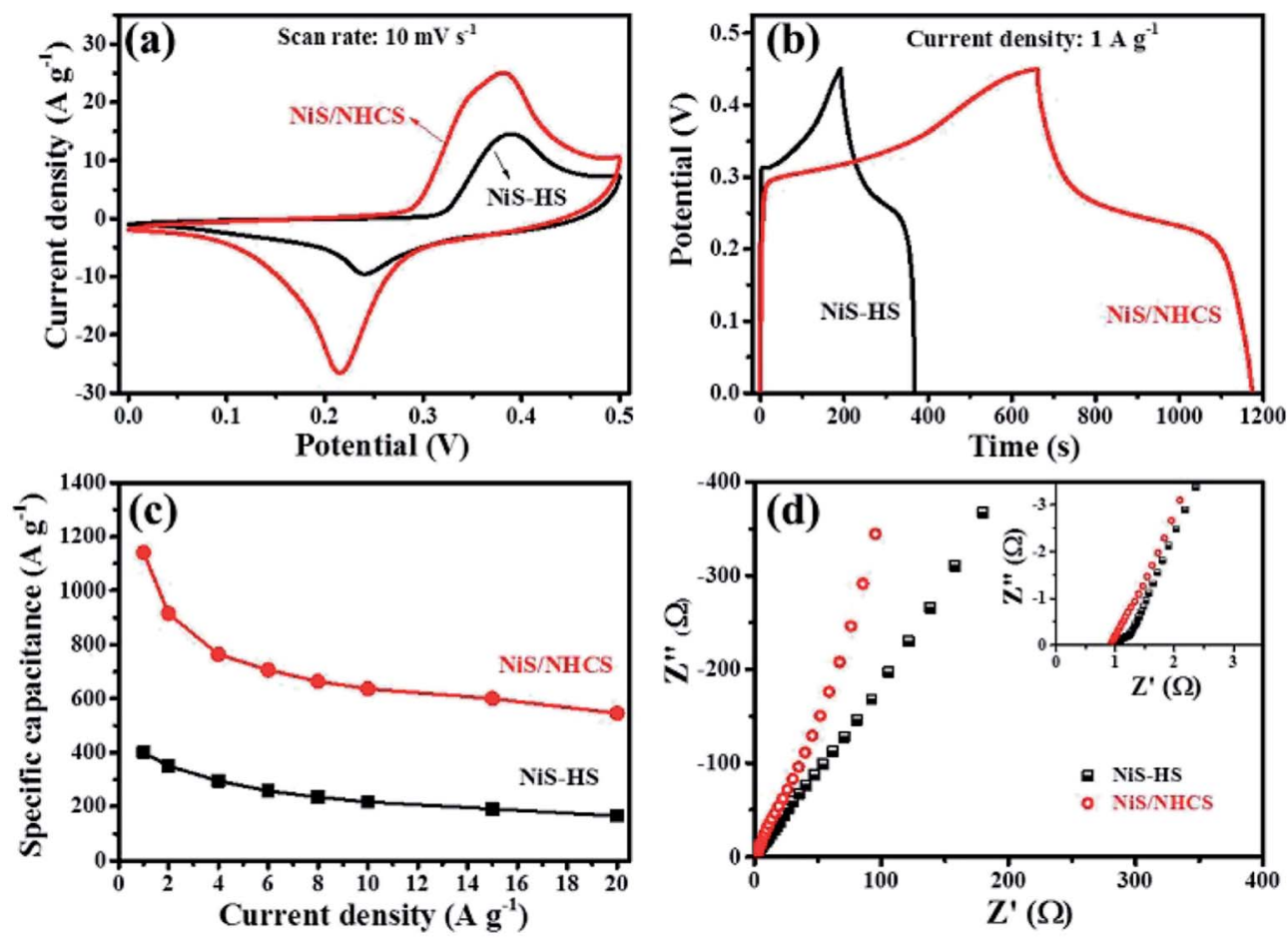

Fig. 5 Cyclic voltammetry curves (a), GCD curves (b), specific capacitance at various current densities (c) and Nyquist plots (d) of NiS/NHCS and $\mathrm{NiS}-\mathrm{HS}$ electrodes. 
and $\mathrm{Si}$ elements. Particularly, the $\mathrm{N}$ signal confirms the successful introduction of $\mathrm{N}$ into the carbon shell. The $\mathrm{C} 1 \mathrm{~s}$ peak in the high-resolution spectrum (Fig. S4b $\dagger$ ) can be deconvoluted into four peaks at 284.6, 285.6, 287.2 and $289.0 \mathrm{eV}$, corresponding to $\mathrm{sp}^{3}$-hybridized $\mathrm{C}-\mathrm{C} / \mathrm{C}=\mathrm{C}, \mathrm{C}-\mathrm{O} / \mathrm{C}-\mathrm{N}$, $\mathrm{C}=\mathrm{O}$ and $-\mathrm{COO}$ bonds. ${ }^{29}$ The high-resolution $\mathrm{N}$ 1s spectrum (Fig. S4c $\dagger$ ) can be deconvoluted into four peaks at 398.3, 400.0, 400.9 and $403.5 \mathrm{eV}$, corresponding to pyridinic- $\mathrm{N}$, pyrrolic- $\mathrm{N}$, quaternary- $\mathrm{N}$ and $\mathrm{N}$-oxide. The $\mathrm{N}$ element can not only increase the conductivity of carbon materials, but also facilitate electrolyte penetration with a feasible pathway. The survey XPS spectrum of NiS/NHCS (Fig. S5 $\dagger$ ) shows peaks for Ni, S, C, N and $\mathrm{O}$ elements, where the signal for $\mathrm{O}$ can be attributed to the oxygen-containing functional group in the NHCS core and sulfur oxides in the NiS shell. ${ }^{33,35,37}$ The high-resolution XPS spectrum of Ni 2p (Fig. 4c) shows two characteristic peaks at 852.1 and $869.7 \mathrm{eV}$ and two shake-up satellites at 858.0 and $876.1 \mathrm{eV}$, which can be attributed to $\mathrm{Ni} 2 \mathrm{p}_{3 / 2}$ and $\mathrm{Ni} 2 \mathrm{p}_{1 / 2}$ orbitals of $\mathrm{Ni}^{2+} \cdot{ }^{36}$ Furthermore, the XPS peak of S 2p (Fig. 4d) is deconvoluted into two binding energy peaks at 162.5 and $163.8 \mathrm{eV}$, which correspond to $\mathrm{S} 2 \mathrm{p}_{3 / 2}$ and $\mathrm{S} 2 \mathrm{p}_{1 / 2}$ orbitals of $\mathrm{S}^{2-}$, respectively. Another peak at $168.3 \mathrm{eV}$ belongs to sulfur oxide from the oxidation of $\mathrm{S}$ with residual $\mathrm{O}_{2} \cdot{ }^{37,38}$

The electrochemical performance of NiS/NHCS and NiS-HS was investigated by using a three-electrode configuration in
2.0 M KOH aqueous solution. The cyclic voltammetry (CV) curves of both samples (Fig. 5a) exhibit a couple of faradaic redox peaks, which can be assigned to the reversible faradaic redox process of $\mathrm{Ni}^{3+} / \mathrm{Ni}^{2+} .{ }^{29}$ The high-resolution XPS spectra of $\mathrm{Ni} 2 \mathrm{p}$ for the NiS/NHCS after charging was used to prove the $\mathrm{Ni}$ valence change in the charging/discharging process. The two evident peaks appearing at 854.8 and $872.4 \mathrm{eV}$ can be ascribed to $\mathrm{Ni}^{3+}$, indicating the occurrence of the redox reaction. It is obviously seen that the integrated CV area of NiS/NHCS is much larger than that of NiS-HS, indicating that NiS/NHCS possesses better capacitive properties. In the galvanostatic charge/ discharge (GCD) curve of NiS/NHCS (Fig. 5b), the discharging time matches well with the charging time, revealing a high reversibility of the pseudocapacitive reaction at the surface of the electrodes. And the potential plateaus correspond to the redox peaks of the $\mathrm{CV}$ curves. Notably, the discharge time of NiS/NHCS is much longer than that of NiS-HS, indicating the markedly improved specific capacitance due to the introduction of NHCSs as the conductive substrate.

The GCD curves of NiS/NHCS and NiS-HS electrodes were measured at various current densities (Fig. S7a and $\mathrm{c} \dagger$ ) to evaluate the rate performance (Fig. 5c). The NiS/NHCS electrode outperforms NiS-HS over the entire current density range tested. For example, the NiS/NHCS electrode shows a high specific capacitance of $1150 \mathrm{~F} \mathrm{~g}^{-1}$ at $1 \mathrm{~A} \mathrm{~g}^{-1}$ and a capacitance

\section{(a)}

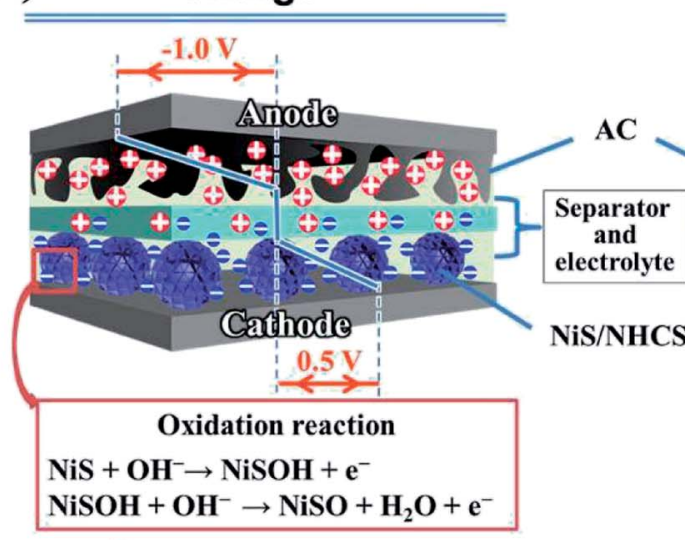

Charge

Discharge

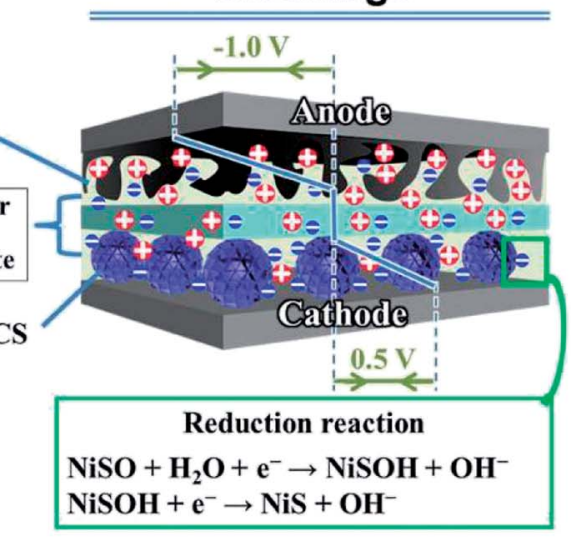

Note: 3 : Cation $\left(\mathrm{K}^{+}\right)$; $\odot$ : Anoin $\left(\mathrm{OH}^{-}\right)$
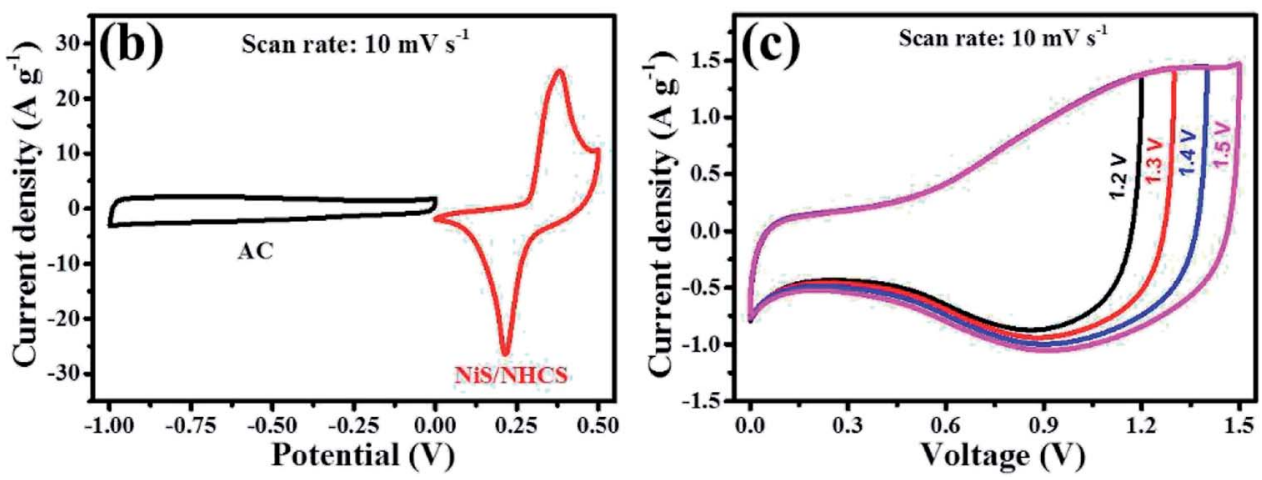

Fig. 6 (a) Configuration and illustration of the energy storage mechanism for the NiS/NHCS\|AC device. (b) CV curves of AC and NiS/NHCS from -1.0 to $0 \mathrm{~V}$ and 0 to $0.5 \mathrm{~V}$, respectively, at $10 \mathrm{mV} \mathrm{s}^{-1}$ in a three-electrode system. (c) CV curves of the as-fabricated NiS/NHCS\|AC device in various operation voltages at a scan rate of $10 \mathrm{mV} \mathrm{s}^{-1}$. 
retention rate of $52.2 \%$ at the high current density of $20 \mathrm{~A} \mathrm{~g}^{-1}$, which are much higher than those of the NiS-HS electrode $(400$ $\mathrm{F} \mathrm{g}^{-1}$ at $1 \mathrm{~A} \mathrm{~g}^{-1}$ and $45.6 \%$ at $20 \mathrm{~A} \mathrm{~g}^{-1}$ ). The $\mathrm{N}$-doped carbon hollow core uniformly deposited with the NiS nanosheets can promote the electron transfer in the NiS/NHCS electrode, leading to enhanced rate performance. The mechanism for the excellent capacitive properties of the NiS/NHCS electrode was further investigated by electrochemical impedance spectroscopy (Fig. 5d). In the high frequency region, there is a pseudosemicircle in the Nyquist plot of NiS-HS while the semicircle is negligible for NiS/NHCS, indicating that NiS/NHCS exhibits a smaller charge-transfer resistance than NiS-HS. In the low frequency region, the slope for the NiS/NHCS electrode is larger than that for NiS-HS, suggesting that NiS/NHCS not only possesses lower diffusive resistance but offers faster ion transport. To evaluate the durability of the electrode materials, the long-term cycling performance of NiS/NHCS and NiS-HS were tested by GCD measurements up to 4000 cycles at $5 \mathrm{~A} \mathrm{~g}^{-1}$. 76\% of the specific capacitance of NiS/NHCS was retained after 4000 cycles compared to $63 \%$ retention for NiS-HS (Fig. S8†).

To further evaluate the NiS/NHCS electrode for practical application, a hybrid supercapacitor (HSC) device was assembled using the NiS/NHCS electrode as the cathode and the active carbon (AC) electrode as the anode in $\mathrm{KOH}$ aqueous electrolyte (Fig. 6a). Fig. 6b shows the CV curves of NiS/NHCS and AC electrodes at a scan rate of $10 \mathrm{mV} \mathrm{s}^{-1}$ with a stable potential range of -1 to $0 \mathrm{~V}$ and 0 to $0.5 \mathrm{~V}$ in the three-electrode system, respectively. The energy storage mechanism of the NiS/ NHCS||AC device is depicted in Fig. 6a. The NiS/NHCS cathode can store energy by an oxidation reaction of $\mathrm{NiS}$ with the incorporation of $\mathrm{OH}^{-}$during the charging process while the AC anode can accumulate electrons by cation (e.g. $\left.\mathrm{K}^{+}\right)$adsorption. ${ }^{39}$ In the charging process, the NiS/NHCS cathode and AC anode can produce an electric potential from 0 to $0.5 \mathrm{~V}$ and from -1 to $0 \mathrm{~V}$, respectively, endowing the HSC device with a broad operating voltage window of $1.5 \mathrm{~V}$. In the discharging process, the NiS/NHCS cathode and AC anode can release energy via the reduction reaction and desorption of ions. The employed AC electrode exhibits a specific capacitance of $280 \mathrm{~F} \mathrm{~g}^{-1}$ at the current density of $1 \mathrm{~A} \mathrm{~g}^{-1}$ (Fig. S9†). According to the charge balance theory between the anode and cathode in the HSC device, the optimal mass loading ratio for the NiS/NHCS and the AC electrode is $m_{(\mathrm{NiS} / \mathrm{NHCS})}: m_{(\mathrm{AC})}=0.54: 1$, while that for the NiS-HS and the AC electrode is $m_{\text {(NiS-HS })}: m_{(\mathrm{AC})}=1.4: 1$. Utilizing the different potential window of the NiS/NHCS and the AC electrode, the operating voltage of the fabricated HSC device is expected to be $1.5 \mathrm{~V}$ (Fig. $6 \mathrm{c}$ and d).

As depicted in Fig. 7a and b, the integrated area of the CV curve of NiS/NHCS||AC was much larger than that of NiS$\mathrm{HS} \mid$ |AC, while the discharging time of the former is also much and longer than that of the latter, indicating that the specific capacitance of NiS/NHCS $\mid$ AC is much larger than that of NiSHS||AC (Fig. 7c). The specific capacitance of the NiS/NHCS||AC exhibited a maximum specific capacitance of $120 \mathrm{~F} \mathrm{~g}^{-1}$ at the
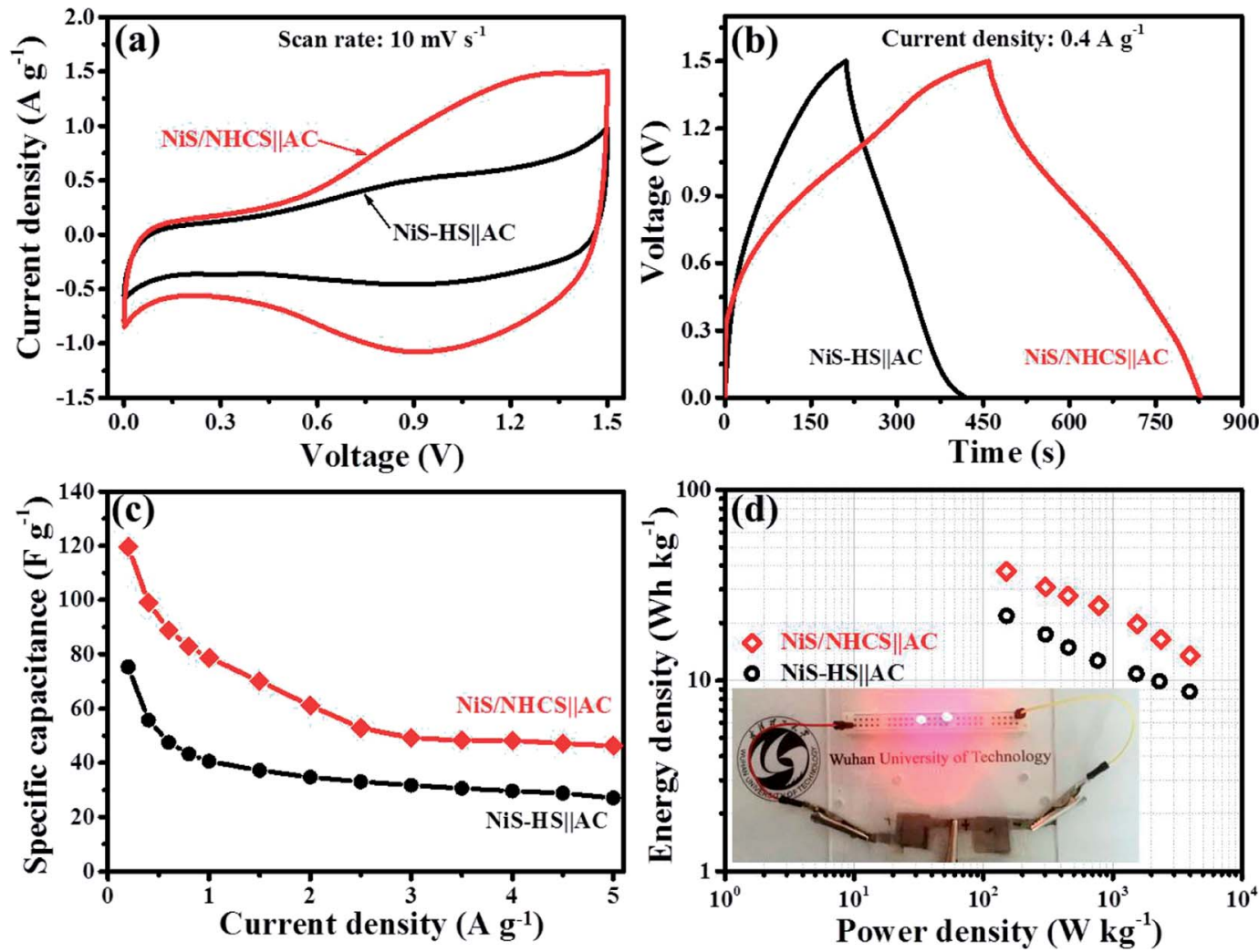

Fig. 7 (a) CV curves, (b) GCD curves, (c) current-density dependence of the specific capacitance and (d) Ragone plots of NiS/NHCSIIAC and NiSHS\|AC. The inset of part d shows two LEDs powered by two NiS/NHCS\|AC devices in series. 
current density of $0.2 \mathrm{~A} \mathrm{~g}^{-1}$ and maintained a capacitance of 46 $\mathrm{F} \mathrm{g}^{-1}$ at $5 \mathrm{~A} \mathrm{~g}^{-1}$, while those of NiS-HS||AC were $75.3 \mathrm{~F} \mathrm{~g}^{-1}$ at $0.2 \mathrm{~A} \mathrm{~g}^{-1}$ and $28.1 \mathrm{~F} \mathrm{~g}^{-1}$ at $5 \mathrm{~A} \mathrm{~g}^{-1}$. In contrast to the single NiS/ NHCS electrode in the three-electrode system, the GCD curves of the HSC devices (Fig. S10a and S11c $\dagger$ ) exhibited a better linear feature, demonstrating that the electron release from the NiS/NHCS redox reaction in the cathode has been well matched with the electron accumulation by cation adsorption in the AC anode.

Energy density and power density are two important factors for evaluating the electrochemical performance for a hybrid supercapacitor device. It can be observed from the Ragone plot (Fig. 7d) that the NiS/NHCS||AC device demonstrates a high energy density of $38.3 \mathrm{~W} \mathrm{~h} \mathrm{~kg}^{-1}$ at a power density of $160 \mathrm{~W} \mathrm{~kg}^{-1}$ with a charging time of $1150 \mathrm{~s}$ (Fig. S10a†). It is worth noting that, even when charging for only $25 \mathrm{~s}$, the HSC can still possess an energy density of $13.5 \mathrm{~W} \mathrm{~h} \mathrm{~kg}^{-1}$ at a high power density of $4000 \mathrm{~W} \mathrm{~kg}^{-1}$. By contrast, the NiS-HS||AC device shows energy densities of $24.8 \mathrm{~W} \mathrm{~h} \mathrm{~kg}^{-1}$ at $150 \mathrm{~W} \mathrm{~kg}^{-1}$ and $8.8 \mathrm{~W} \mathrm{~h} \mathrm{~kg}^{-1}$ at $3944 \mathrm{~W} \mathrm{~kg}^{-1}$. To manifest the practical operating performance of the NiS/NHCS||AC device, two LEDs (with lowest working voltage of $\sim 2.2 \mathrm{~V}$ ) can be powered by two HSC devices connected in series $(\sim 1.4 \mathrm{mg}$ active materials in total for each), with red light emission lasting for 110 s. Fig. S12 $\uparrow$ shows the long-term cycling property of the NiS/NHCS||AC at a current density of $3 \mathrm{~A} \mathrm{~g}^{-1}$. Remarkably, the NiS/NHCS||AC exhibited excellent cycling stability and still delivered $96 \%$ of its initial capacitance after 5000 cycles. After 5000 cycles, NiS nanosheets were still anchored on the surface of NHCSs (Fig. S13 $\dagger$ ), indicating that the NiS/NHCS composite possessed a stable chemical structure.

The excellent supercapacitive performance of NiS/NHCS can be attributed to several aspects. On the one hand, the NiS/NHCS composite with a novel hierarchical architecture can facilitate the diffusion of electrolyte ions and supply sufficient electroactive sites for redox reactions. On the other hand, the NHCS core can markedly increase the electron transfer efficiency in the electrode material and alleviate the aggregation of NiS nanosheets during the charge/discharge process. ${ }^{\mathbf{4 0 - 4 2}}$ Finally, the NHCSs as the backbone could support the NiS nanosheets stably during the charge/discharge process, which is beneficial for the cycling stability. Hence, the NiS/NHCS composite can be regarded as a promising candidate material for highperformance supercapacitors, due to its high specific capacitance and outstanding stability.

\section{Conclusion}

A hierarchical NiS/NHCS composite with enhanced supercapacitive performance can be rationally designed and fabricated by a sacrificial template method. The NiS/NHCS composite exhibits a specific capacitance of $1150 \mathrm{~F} \mathrm{~g}^{-1}$ at $1 \mathrm{Ag}^{-1}$ and $600 \mathrm{~F} \mathrm{~g}^{-1}$ at the high current density of $20 \mathrm{Ag}^{-1}$. And the hybrid supercapacitor device assembled with NiS/NHCS as the cathode and an activated carbon electrode as the anode delivers a high energy density of $38.3 \mathrm{~W} \mathrm{~h} \mathrm{~kg}^{-1}$ at a power density of $160 \mathrm{~W} \mathrm{~kg}^{-1}$ and has an excellent cycle performance with the retention rate of $96 \%$ after 5000 cycles. Moreover, the strategy reported herein can be applied for synthesizing other hierarchical carbon-metal sulfide composite materials for energy conversion and storage applications.

\section{Experimental section}

\section{Synthesis of $\mathrm{N}$-doped carbon $/ \mathrm{SiO}_{2}$ spheres}

$\mathrm{N}$-doped carbon/SiO ${ }_{2}\left(\mathrm{~N}\right.$-carbon/SiO $\left.{ }_{2}\right)$ spheres were synthesized by a facile one-step method involving the formation of spherical $\mathrm{SiO}_{2}$ cores and the coating of polydopamine. Briefly, a mixture containing $12 \mathrm{~mL}$ of anhydrous ethanol, $0.5 \mathrm{~mL}$ of ammonia $(25 \%)$ and $40 \mathrm{~mL}$ of distilled water was vigorously stirred for $10 \mathrm{~min}$. One milliliter of tetraethoxysilane (TEOS) was added to the mixture dropwise, and the stirring continued for an additional $15 \mathrm{~min}$. Subsequently, $4 \mathrm{~mL}$ of dopamine hydrochloride (DA) aqueous solution (50 $\mathrm{mg} \mathrm{mL}^{-1}$ ) was added to the above solution and kept stirring for $32 \mathrm{~h}$. Brown polydopamine $/ \mathrm{SiO}_{2}$ $\left(\mathrm{PDA} / \mathrm{SiO}_{2}\right)$ spheres were collected after centrifugation and washed with purified water three times. $\mathrm{N}$-carbon $/ \mathrm{SiO}_{2}$ spheres were obtained after calcination at $800{ }^{\circ} \mathrm{C}$ for $2 \mathrm{~h}$ under a nitrogen $\left(\mathrm{N}_{2}\right)$ atmosphere.

\section{Synthesis of the NiSi/NHCS composite}

For preparation of NiSi/NHCS, $40 \mathrm{mg}$ of $\mathrm{N}$-carbon/ $/ \mathrm{SiO}_{2}$ was dispersed in $40 \mathrm{~mL}$ of water by sonication for $0.5 \mathrm{~h}$, followed by the addition of $\mathrm{NiCl}_{2}(0.5 \mathrm{mmol}), \mathrm{NH}_{4} \mathrm{Cl}(5 \mathrm{mmol})$ and $\mathrm{NH}_{3} \cdot \mathrm{H}_{2} \mathrm{O}$ $(0.5 \mathrm{~mL})$ under mild stirring. After stirring for $0.5 \mathrm{~h}$, the mixture suspension was transferred into a Teflon-lined autoclave and maintained at $105{ }^{\circ} \mathrm{C}$. After reaction for $2 \mathrm{~h}$ and $12 \mathrm{~h}$, $\mathrm{NiSi} / \mathrm{NHCS} / \mathrm{SiO}_{2}$ and $\mathrm{NiSi} / \mathrm{NHCS}$ composites were obtained, respectively.

\section{Synthesis of NiS/NHCS}

$40 \mathrm{mg}$ of NiSi/NHCS and $100 \mathrm{mg}$ of polyvinylpyrrolidone (PVP, $\mathrm{K}-30$ ) were added to $60 \mathrm{~mL}$ of distilled water and sonicated for $0.5 \mathrm{~h}$. Subsequently, $600 \mathrm{mg}$ of $\mathrm{Na}_{2} \mathrm{~S} \cdot 9 \mathrm{H}_{2} \mathrm{O}$ was added during stirring, and the suspension was transferred to a $100 \mathrm{~mL}$ Teflonlined autoclave and heated at $160{ }^{\circ} \mathrm{C}$ for $12 \mathrm{~h}$. The product was obtained after washing with purified water and ethanol several times and annealing at $300{ }^{\circ} \mathrm{C}$ for $2 \mathrm{~h}$ in a $\mathrm{N}_{2}$ atmosphere. For comparison, NiS hollow spheres (NiS-HS) were fabricated under the same conditions as those of the synthesis of NiS/NHCS, except that $\mathrm{SiO}_{2}$ spheres were used instead of the N-carbon/ $\mathrm{SiO}_{2}$.

\section{Materials characterization}

Powder X-ray diffraction (XRD) patterns of the samples were recorded on a Rigaku X-ray diffractometer with $\mathrm{Cu} \mathrm{K}_{\alpha}$ radiation $(\lambda=0.15418 \mathrm{~nm})$. The morphologies were examined with a field emission scanning electron microscope (FESEM, JSM-7500) and transmission electron microscope (TEM, Titan G2). Nitrogen adsorption-desorption experiments were performed on a Micromeritics ASAP 2020 nitrogen adsorption apparatus to measure the specific surface area and porosity of the samples. The X-ray photoelectron spectroscopy (XPS) was conducted on a Thermo ESCALAB 250. Raman spectrum analysis was 
performed by using a Renishaw Raman microscope under $\lambda_{\text {exc }}=$ $633 \mathrm{~nm}$ laser excitation.

\section{Electrochemical measurements}

The working electrodes were obtained by brushing a mixture of active materials (NiS/NHCS, NiS-HS or AC), acetylene black and polyvinylidene fluoride (mass ratio $8: 1: 1$ ) in N-methyl pyrrolidone (NMP) solvent onto nickel foam and air-drying at $100{ }^{\circ} \mathrm{C}$ overnight. The mass loading of the active material for individual electrodes was $1.0 \mathrm{mg} \mathrm{cm}^{-2}$. A three-electrode system configuration was used to test the electrochemical performance of individual electrodes, where a platinum foil served as the counter electrode and a $\mathrm{Ag} / \mathrm{AgCl}$ electrode served as the reference electrode. Cyclic voltammetry (CV), galvanostatic charge/ discharge (GCD) and electrochemical impedance spectroscopy (EIS) were carried out on a electrochemical workstation (CHI $760 \mathrm{E}$ ) in $2.0 \mathrm{M} \mathrm{KOH}$ electrolyte. Moreover, the ACS device was assembled with NiS/NHCS as the cathode and AC as the anode to measure its potential for practical application.

\section{Conflicts of interest}

There are no conflicts to declare.

\section{Acknowledgements}

This work was supported by the NSFC (21433007, 51372190, 21573170 and 51320105001), 973 program (2013CB632402), NSFHP (2015CFA001), Innovative Research Funds of SKLWUT (2017-ZD-4) and Fundamental Research Funds of Central Universities (WUT: 2015-III-034).

\section{Notes and references}

1 B. You and Y. Sun, Adv. Energy Mater., 2016, 6, 1502333.

2 J. Low, J. Yu and W. Ho, J. Phys. Chem. Lett., 2015, 6, 42444251.

3 J. Zhang, H. Hu, Z. Li and X. W. Lou, Angew. Chem., Int. Ed., 2016, 55, 3982-3986.

4 T. Liu, C. Jiang, W. You and J. Yu, J. Mater. Chem. A, 2017, 5, 8635-8643.

5 Z. N. Yu, L. Tetard, L. Zhai and J. Thomas, Energy Environ. Sci., 2015, 8, 702-730.

6 J. Jiang, Y. Li, J. Liu, X. Huang, C. Yuan and X. W. Lou, Adv. Mater., 2012, 24, 5166-5180.

7 Z. S. Wu, Y. Sun, Y. Z. Tan, S. Yang, X. Feng and K. Mullen, J. Am. Chem. Soc., 2012, 134, 19532-19535.

8 X. Rui, H. Tan and Q. Yan, Nanoscale, 2014, 6, 9889-9924.

9 M. Sevilla and R. Mokaya, Energy Environ. Sci., 2014, 7, 12501280.

10 W. Xia, A. Mahmood, R. Zou and Q. Xu, Energy Environ. Sci., 2015, 8, 1837-1866.

11 W. Zhou, X. Cao, Z. Zeng, W. Shi, Y. Zhu, Q. Yan, H. Liu, J. Wang and H. Zhang, Energy Environ. Sci., 2013, 6, 22162221.
12 C. Sun, M. Ma, J. Yang, Y. Zhang, P. Chen, W. Huang and X. Dong, Sci. Rep., 2014, 4, 7054.

13 W. Li, S. Wang, L. Xin, M. Wu and X. Lou, J. Mater. Chem. A, 2016, 4, 7700-7709.

14 Z. Li, J. Han, L. Fan and R. Guo, CrystEngComm, 2015, 17, 1952-1958.

15 G. Liu, B. Wang, L. Wang, T. Liu, T. Gao and D. Wang, RSC Adv., 2016, 6, 54076-54086.

16 S. C. Riha, A. A. Koegel, X. Meng, I. S. Kim, Y. Cao, M. J. Pellin, J. W. Elam and A. B. Martinson, ACS Appl. Mater. Interfaces, 2016, 8, 2774-2780.

17 Z. Ai, Z. Hu, Y. Liu, M. Fan and P. Liu, New J. Chem., 2016, 40, 340-347.

18 Y. M. Chen, Z. Li and X. W. Lou, Angew. Chem., Int. Ed., 2015, 54, 10521-10524.

19 X. Yu, L. Yu, L. Shen, X. Song, H. Chen and X. W. Lou, Adv. Funct. Mater., 2014, 24, 7440-7446.

20 J. Yang, W. Guo, D. Li, C. Wei, H. Fan, L. Wu and W. Zheng, J. Power Sources, 2014, 268, 113-120.

21 B. Zhu, Z. Wang, S. Ding, J. Chen and X. W. Lou, RSC Adv., 2011, 1, 397-400.

22 X. Cai, R. V. Hansen, L. Zhang, B. Li, C. K. Poh, S. H. Lim, L. Chen, J. Yang, L. Lai, J. Lin and Z. Shen, J. Mater. Chem. A, 2015, 3, 22043-22052.

23 J. Yang, C. Yu, X. Fan, S. Liang, S. Li, H. Huang, Z. Ling, C. Hao and J. Qiu, Energy Environ. Sci., 2016, 9, 12991307.

24 Z. Lei, J. Zhang, L. Zhang, N. A. Kumar and X. Zhao, Energy Environ. Sci., 2016, 9, 1891-1930.

$25 \mathrm{H}$. Li, Y. Tao, X. Zheng, J. Luo, F. Kang, H. Cheng and Q. Yang, Energy Environ. Sci., 2016, 9, 3135-3142.

26 P. R. Jothi, R. R. Salunkhe, M. Pramanik, S. Kannan and Y. Yamauchi, RSC Adv., 2016, 6, 21246-21253.

27 L. Chen, Y. Lu, L. Yu and X. W. Lou, Energy Environ. Sci., 2017, 10, 1777-1783.

28 G. P. Pandey, T. Liu, E. Brown, Y. Yang, Y. Li, X. Sun, Y. Fang and J. Li, ACS Appl. Mater. Interfaces, 2016, 8, 9200-9210.

29 F. Pei, T. An, J. Zang, X. Zhao, X. Fang, M. Zheng, Q. Dong and N. Zheng, Adv. Energy Mater., 2016, 6, 1502539.

30 Y. Wang, G. Wang, H. Wang, C. Liang, W. Cai and L. Zhang, Chem.-Eur. J., 2010, 16, 3497-3503.

31 K. S. W. Sing, D. H. Everett, R. A. W. Haul, L. Moscou, R. A. Pierotti, J. Rouquérol and T. Siemieniewska, Pure Appl. Chem., 1985, 57, 603-619.

32 J. Fu, B. Zhu, C. Jiang, B. Cheng, W. You and J. Yu, Small, 2017, 13, 1603938.

33 S. Gao, G. Li, Y. Liu, H. Chen, L. Feng, Y. Wang, M. Yang, D. Wang, S. Wang and X. Zou, Nanoscale, 2015, 7, 23062316.

34 J. Yang, M. Ma, C. Sun, Y. Zhang, W. Huang and X. Dong, J. Mater. Chem. A, 2015, 3, 1258-1264.

35 T. Peng, H. Yi, P. Sun, Y. Jing, R. Wang, H. Wang and X. Wang, J. Mater. Chem. A, 2016, 4, 8888-8897.

36 Y. Zhang, L. Zuo, L. Zhang, J. Yan, H. Lu, W. Fan and T. Liu, Nano Res., 2016, 9, 2747-2759.

37 Y. Li, K. Ye, K. Cheng, J. Yin, D. Cao and G. Wang, J. Power Sources, 2015, 274, 943-950. 
38 J. Chen, X. Wang, J. Wang and P. S. Lee, Adv. Energy Mater., 2016, 6, 1501745.

39 F. Wang, S. Xiao, Y. Hou, C. Hu, L. Liu and Y. Wu, RSC Adv., 2013, 3, 13059-13084.

40 T. Liu, C. Jiang, B. Cheng, W. You and J. Yu, J. Power Sources, 2017, 359, 371-378.
41 Y. Wang, B. Chen, Z. Chang, X. Wang, F. Wang, L. Zhang, Y. Zhu, L. Fu and Y. Wu, J. Mater. Chem. A, 2017, 5, 89818988.

42 H. Sun, Y. Zhu, B. Yang, Y. Wang, Y. Wu and J. Du, J. Mater. Chem. A, 2016, 4, 12088-12097. 